\title{
Convergence Results for 3D Sparse Grid Approaches
}

\author{
J. Noordmans and P. W. Hemker*
}

CWI, Kruislaan 413, 1098 SJ Amsterdam, The Netherlands

\begin{abstract}
The convergence behaviour of solution algorithms is investigated for the anisotropic Poisson problem on partially ordered, sparse families of regular grids in 3D. In order to study multilevel techniques on sparse families of grids, first we consider the convergence of a two-level algorithm that applies semi-coarsening successively in each of the coordinate directions. This algorithm shows good convergence, but recursive application of the successive semi-coarsening is not sufficiently efficient. Therefore we introduce another algorithm, which uses collective 3D semi-coarsened coarse grid corrections. The convergence behaviour of this collective version is worse, due to the lack of correspondence between the solutions on the different grids. By solving for the trivial solution we demonstrate that a good convergence behaviour of the collective version of the algorithm can be retained when the different solutions are sufficiently coherent. In order to solve also non-trivial problems, we develop a defect correction process. This algorithm makes use of hierarchical smoothing in order to deal with the problems related to the lack of coherence between the solutions on the different grids. Now good convergence rates are obtained also for non-trivial solutions. All convergence results are obtained for two-level processes. The results show convergence rates which are bounded, independent of the discretisation level and of the anisotropy in the problem. (c) $1998 \mathrm{John}$ Wiley \& Sons, Ltd.
\end{abstract}

KEY WORDS convergence; multigrid; sparse grids

\section{Introduction}

Classical multigrid (MG) uses sequentially nested families of grids. In the regular, $d$ dimensional case every coarse grid-cell is formed by combination of $2^{d}$ finer grid cells. For more dimensions, as $d$ becomes larger, this implies that many frequencies that can be represented on a fine grid cannot be seen on the next coarser one. Since the two basic mechanisms in an MG method are smoothing and coarse-grid correction, this means that, for higher dimensions (e.g., $d=3$ ), very strong relaxation techniques are required to obtain good convergence results. A way to simplify this heavy requirement is to use semi-coarsening, i.e., a coarser grid is created by only combining a pair of grid cells to a

* Correspondence to P. W. Hemker, CWI, Kruislaan 413, 1098 SJ Amsterdam, The Netherlands. 
coarser grid cell. Obviously, this process of semi-coarsening can be applied in each of the $d$ co-ordinate directions. Subsequent application of this process in all co-ordinate directions makes a partially ordered set of grids rather than a sequentially ordered one. All grids that can be seen as the (semi-) coarsenings of one particular grid are called a full family of grids. Semi-coarsening has been earlier described for $d=2$ in $[16,17]$ and for $d=3$ in [1-3, 11-15].

It is an additional disadvantage of regular refinement in each direction, especially for higher dimensions, that the number of the degrees of freedom increases very fast when more levels of refinement are introduced. In the battle against the increasing number of degrees of freedom, a promising development is the sparse grid approach of Zenger [1$11,13-15,18,19,21]$. Sparse grids are formed by taking a subset from the partially ordered family of semi-refined grids. This subset, the sparse family of grids, is formed by taking all grids for which the cells exceed a certain specified volume. For sufficiently smooth functions it can be shown that the representation of a function on a sparse grid can be much more efficient than on a regular full grid $[13,21]$. Results on which this statement is based are summarized in Section 2.

The problem with a representation on a sparse family of grids is that there is no unique finest grid. There is a whole set of finest grids and the question arises of how to represent a final solution. Basically there are two ways to answer the question. The first is to introduce hierarchical basis functions instead of standard basis functions [1-3,12,21]. In this way one has to add the contributions (the hierarchical surplus) from all grids in the sparse family to form the final solution. The second method is based on extrapolation. Then a linear combination of the approximations on the finest grids is formed. This is called the combination technique $[6,7,11]$.

An advantage of the hierarchical basis (HB) technique is the existence of a straightforward and unique representation of an approximation, and the possibility to write down the Finite Element (FE) discretization for a differential equation. It is a disadvantage that the FE discrete operator is not sparse and that-for variable coefficient equations-no efficient method is available for its evaluation without serious modification of the FE method.

Notice that the solution of a sparse grid problem on an $\mathrm{HB}$ is much different from the usual full grid problem on an $\mathrm{HB}$, where a sequential ordering of grids is used as discussed, e.g., in [20]. In the sparse grid case there exists a partial ordering between the approximations on the different grids, but the approximations do not form a lattice (i.e., no finest grid is available), and no simple transformation to a standard basis exists.

It should be mentioned, however, that the standard hierarchical basis functions method can be implemented by means of a simple transformation from the standard hierarchical to the standard basis functions [11]. In this way the residuals can be computed from the sparse standard discretization matrix. On the other hand, the preconditioner can be based on the hierarchical basis functions matrix. Furthermore, the $p$-version of the hierarchical basis functions allows arbitrary high orders of approximation and, consequently, if the solution is sufficiently smooth, very sparse grids can be used compared with the use of piecewise linear approximations.

In contrast to the hierarchical basis technique, the combination technique makes use of standard basis FE solutions on the finest grids. This has the advantage that existing techniques can be applied for the solution process on these grids.

The above arguments are the motivation to develop methods which use 3D MG semicoarsening techniques together with a sparse family of grids. The combination should also combine the advantages of both approaches, i.e., MG convergence rates should be obtained 
without the requirement of strong relaxation techniques, and the solution of the problem can be represented with sufficient accuracy by a relatively small number of degrees of freedom. An additional advantage can be that the method is insensitive for anisotropies in the problem.

Before we study the solution of discrete equations in Sections 3-5, we first give a summary of approximation results to show the advantage of sparse grid over regular grid approximation.

\section{Approximation on sparse grids}

The advantage of sparse grids in general is the efficient representation of functions, i.e., the possibility to obtain a high accuracy for a relatively small number of coefficients (degrees of freedom). Although sparse grids can also be applied with piecewise polynomials of higher degree [4], we restrict ourselves here to piecewise $d$-linear approximations because solution methods for these simpler methods will lead the way to preconditioning of the more accurate ones.

Of course, the ability to accurately approximate functions with a limited number of degrees of freedom, depends highly on the smoothness of the functions approximated. Whereas in the classical approximations methods usually $C^{n}(\Omega)$ or $H^{n}(\Omega)$ functions are studied, where all derivatives up to an order $n$ are equally involved, for sparse grid approximations this is not the case. Here, mixed derivatives play a special role. Therefore special norms are introduced for the derivation of error bounds.

To illustrate the use of sparse grids in function approximation we introduce some special (semi-)norms and with these norms in the upper bounds we express the main approximation properties. Many results of this type can be found in the literature on sparse grids (loc. cit.), but they are dispersed and generally their formulation is not quite accessible. Therefore, for reference, we summarize here some results for which the proofs can be found in in [13]. For comparison we also give results for regular grids.

\subsection{Notation}

To precisely formulate the approximation results, we first have to summarize some necessary notation.

- Domain $\Omega \subset \mathbb{R}^{d}$, with co-ordinates $x_{j}, j=1, \cdots, d$.

- Multi-integer: $\mathbf{m}=\left(m_{1}, m_{2}, \cdots . m_{d}\right)$,

$$
\begin{aligned}
& -\mathbf{0}=(0,0, \cdots, 0), \quad-\mathbf{1}=(1,1, \cdots, 1) \text {, } \\
& -|\mathbf{m}|=\sum_{j=1}^{d} m_{j}, \quad-\lceil\mathbf{m}\rceil=\max _{j=1, \cdots, d} m_{j}, \\
& \text { - }\|\mathbf{m}\|=\sqrt{\sum_{j=1}^{d} m_{j}^{2}}, \quad-\quad\|\mathbf{m}\|=\prod_{j=1}^{d} m_{j} \text {. }
\end{aligned}
$$

- Dyadic grid $\Omega_{\mathbf{n}}$, grid with dyadic mesh width $\mathbf{h}_{\mathbf{k}}$,

- Mesh width: $\mathbf{h} \in \mathbb{R}^{d} ; \mathbf{h}=\left(h_{1}, h_{2}, \cdots, h_{d}\right)$,

- Mesh size: $\|\mathbf{h}\|=\sqrt{\sum_{i=1}^{d} h_{i}^{2}}$,

- Mesh volume: $\|\mathbf{h}\|=\prod_{i=1}^{d} h_{i}$,

- Dyadic mesh width: $\mathbf{h}_{\mathbf{k}}=\left(h_{k_{1}}, h_{k_{2}}, \cdots, h_{k_{d}}\right)$ with $h_{k_{i}}=2^{-k_{i}}$. 
- Derivatives: $D^{\mathrm{m}}=\prod_{j=1}^{d} \frac{\partial^{m_{j}}}{\partial x_{j}}$.

- Hat function: $\varphi(x)=\max (0,1-|x|)$.

- Basis functions: $\varphi_{\mathbf{n j}} \mathbf{( x )}=\prod_{i=1}^{d} \varphi\left(x_{i} / h_{k_{i}}-j_{i}\right)$.

- Space of piecewise $d$-linear functions on $\Omega_{\mathbf{n}}: V_{\mathbf{n}}=\operatorname{span}\left\{\varphi_{\mathbf{n j}} \mid \mathbf{0} \leq \mathbf{j} \leq 2^{\mathbf{n}}\right\}$.

- Space of hierarchical surpluses on $\Omega_{\mathbf{n}}: W_{\mathbf{n}}=\operatorname{span}\left\{\varphi_{\mathbf{n} \mathbf{j}} \mid \mathbf{0} \leq \mathbf{j} \leq 2^{\mathbf{n}}\right.$, $\|\mathbf{j}\|$ odd $\}$.

- Norms on the function spaces, $p \geq 1,0 \leq \ell \leq d$,

- $\|u\|_{\infty}=\|u\|_{L_{\infty}}=\operatorname{supess}_{x \in \Omega}|u(\mathbf{x})|$,

$-\|u\|_{p}=\|u\|_{L_{p}}=\left(\int_{\Omega}|u(\mathbf{x})|^{p} d \Omega\right)^{1 / p}$,

$-\|u\|_{W_{p}^{\mathbf{n}, \ell}}=\left(\sum_{\substack{\mathbf{0} \mathbf{m} \mid=\ell \\ \mathbf{0} \leq \mathbf{m} \leq 1}}\left\|D^{\mathbf{n}+\mathbf{m}_{u}} u\right\|_{p}^{p} d \Omega\right)^{1 / p}$,

- $\|u\|_{W_{p}^{1}}=\|u\|_{W_{p}^{0}}^{0,1}$, the usual first order Sobolev norm.

\subsection{Errors on regular grids}

Let $u_{\mathbf{n}}$ be the pointwise projection at $\cup_{\mathbf{k} \leq \mathbf{n}} \Omega_{\mathbf{k}}=\Omega_{\mathbf{n}}$ of a function with finite norm $\left\|D^{\mathbf{1}+\mathbf{m}} u\right\|_{p}$, with $p=2, \infty$, and $\mathbf{0} \leq \mathbf{m} \leq \mathbf{1}$, on $V_{\mathbf{n}}=\oplus_{\mathbf{k} \leq \mathbf{n}} W_{\mathbf{k}}$. Error bounds for the approximation on regular grids are [13, Theokrem 3.3]:

- $\left\|u-u_{\mathbf{n}}\right\|_{2} \leq 2^{-d} 3^{-3|\mathbf{m}| / 2} \sum_{i=1}^{d} h_{n_{i}}^{\left(1+m_{i}\right)}\left\|D^{\mathbf{1}+\mathbf{m}} u\right\|_{2}$,

- $\left\|u-u_{\mathbf{n}}\right\|_{\infty} \leq 6^{-|\mathbf{m}|} \sum_{i=1}^{d} h_{n_{i}}^{\left(1+m_{i}\right)}\left\|D^{1+\mathbf{m}_{u}} u\right\|_{\infty}$.

It follows that for square grids, for which $\left\|\mathbf{h}_{n \cdot 1}\right\|^{2}=\sum_{i=1}^{d}\left(2^{-n}\right)^{2}=d 2^{-2 n}$, we have:

- $\left\|u-u_{n \cdot 1}\right\|_{2} \leq 3^{-2 d}\left\|\mathbf{h}_{n \cdot 1}\right\|^{2}\left\|D^{2 \cdot 1} u\right\|_{2}$,

- $\left\|u-u_{n \cdot 1}\right\|_{\infty} \leq 6^{-d}\left\|\mathbf{h}_{n \cdot 1}\right\|^{2}\left\|D^{2 \cdot 1} u\right\|_{\infty}$.

By simple counting it is seen that for this approximation the number of degrees of freedom is $\mathcal{O}\left(2^{|\mathbf{n}|}\right)$, and therefore for a square grid $\mathscr{O}\left(2^{n d}\right)$.

\subsection{Errors on sparse grids}

Let $\hat{u}_{n}$ be the pointwise projection at $\bigcup_{|\mathbf{k}| \leq n} \Omega_{\mathbf{k}}=\hat{\Omega}_{n}$ of a function with finite norm $\left\|D^{\mathbf{1}+\mathbf{m}} u\right\|_{p}$, with $p=2, \infty$, and $\mathbf{0} \leq \mathbf{m} \leq \mathbf{1}$, on the sparse grid space $\hat{V}_{n}=\oplus_{|\mathbf{k}| \leq n} W_{\mathbf{k}}$. Error bounds for approximations on these sparse grids [13, Theorem 3.6] are given by:

- $\left\|u-\hat{u}_{n}\right\|_{p} \leq C\|\mathbf{h}\|^{2} \log ^{d-1}\|\mathbf{h}\|^{-1}\left\|D^{2 \cdot 1} u\right\|_{p}$,

- $\left\|u-\hat{u}_{n}\right\|_{p} \leq C\|\| \mathbf{h}\left\|\log ^{d-1-|\mathbf{m}|}\right\| \mathbf{h}\left\|^{-1}\right\| D^{1+\mathbf{m}} u \|_{p}$,

- $\left\|u-\hat{u}_{n}\right\|_{p} \leq C\|\mathbf{h}\|^{1+\ell / d} \log ^{d-1}\|\mathbf{h}\|^{-1}\|u\|_{W_{p}^{1, \ell}}$,

where $\ell$ is any integer, $0 \leq \ell \leq d$. Bounds for the errors on sparse grids in energy norm are [13, Theorem 3.7]:

- $\left\|u-\hat{u}_{n}\right\|_{W_{p}^{1}} \leq C\|\mathbf{h}\| \log ^{d-1}\|\mathbf{h}\|^{-1}\|u\|_{W_{p}^{1,1}}$,

- $\left\|u-\hat{u}_{n}\right\|_{W_{p}^{1}} \leq C\|\mathbf{h}\|\left\|D^{2 \cdot 1} u\right\|_{p}$.

Notice that here $\|\mathbf{h}\|=2^{-|\mathbf{k}|}$ is the volume of the finest cells in the sparse grid.

For this approximation, by counting, we see that the number of degrees of freedom for the sparse grid approximation is $\mathcal{O}\left(2^{n} n^{d-1}\right)$. This number is significantly less than the $\mathcal{O}\left(2^{\text {nd }}\right)$ 
for the regular grid. Therefore, in the remainder of this paper, we study the possibility of finding a solution method to solve a PDE on a sparse grid with an amount of computational operations of $\mathbb{O}\left(2^{n} n^{d-1}\right)$.

\section{The equation}

In the remainder of this paper we study the 3D anisotropic Poisson equation with homogeneous boundary conditions:

$$
-\nabla \cdot(\mathbf{a} \nabla u)=f \quad \text { on } \quad \Omega=[0,1]^{3}
$$

where $\mathbf{a}=\operatorname{diag}\left(a_{11}, a_{22}, a_{33}\right)$ is a constant diagonal tensor. First, we discretize the equation by the usual finite element (FE) technique on the regular grid $\Omega_{\mathbf{k}}$. A standard FE method on grid $\Omega_{\mathbf{k}}$ is obtained by selecting trial and test functions in $B_{\mathbf{k}}=\left\{\varphi_{\mathbf{n j}} \mid \mathbf{0} \leq \mathbf{j} \leq 2^{\mathbf{k}}\right\}$. This yields the discrete equations $\sum_{\mathbf{j}} a\left(\varphi_{\mathbf{k j}}, \varphi_{\mathbf{k i}}\right) u_{\mathbf{k j}}=f\left(\varphi_{\mathbf{k i}}\right)$, which are also denoted in matrix form by

$$
A_{\mathbf{k k}} u_{\mathbf{k}}=f_{\mathbf{k}}
$$

Let $A_{\ell}$ be the block diagonal matrix composed by all stiffness matrices on level $\ell$,

$$
A_{\ell}=\operatorname{diag}\left(A_{\mathbf{k k}}|| \mathbf{k} \mid=k_{1}+k_{2}+k_{3}=\ell\right)
$$

and let $f_{\ell}, u_{\ell}$ be the concatenation of right-hand side vectors $f_{\mathrm{k}}$ and solution vectors $u_{\mathbf{k}}$ respectively. Then the collection of all discretizations on the grids $\Omega_{\mathbf{k}}$ with $|\mathbf{k}|=\ell$ can be written as

$$
A_{\ell} u_{\ell}=f_{\ell}
$$

In this paper we study iteration methods for the solution of (3.3). In (3.3) possibly the FE method can be replaced by another discretization technique. Although we are essentially interested in MG methods, for simplicity in this paper we restrict ourselves to the corresponding two-grid methods.

\section{A successive correction method}

In this section we consider a method for which each cycle consists of three stages, one for each co-ordinate direction $i=1,2,3$. In each stage it uses the one-dimensional (semicoarsened) full approximation scheme (FAS) in direction $i$. We call the method SCM (successive correction method). This method is similar to the one proposed by Pflaum [19] in the sense that each time the coarse-grid correction is obtained only from coarsening in the $i$ th direction. For grids that have no coarser grid in the $i$ th direction we perform only relaxations, in order to have a constant number of relaxations for all the grids on the fine level. For relaxation on the fine level we use Jacobi relaxation with a damping factor $\omega=\frac{1}{2}$. We apply one pre- $\left(\nu_{1}=1\right)$ and one post-relaxation $\left(\nu_{2}=1\right)$ for every cycle.

For convergence measurement we solve the equation (3.1) with homogeneous right hand side, $f=0$. The trivial solution has the advantage of a possible lasting monitoring of the convergence. As an initial guess we use $u_{0}=x(1-x) y(1-y) z(1-z)$. After every $S C M$ cycle we calculate the residual on each grid of the fine level $\ell$. In Figure 3 we see the 
convergence history of the $S C M$ cycling. We repeat the experiment for different (plane and line) anisotropies, viz. $a_{11}=1.0,0.01$ and 100.0. In all cases $a_{22}=a_{33}=1$.

We make the following observations. During convergence we can distinguish two phases: an initial and an asymptotic phase. The asymptotic convergence rate begins after 10 or 20 iterations and appears to be $\rho \approx 0.35$, independent of the grid and independent of the level. Also for the different anisotropies the convergence rates tend towards the same constant value for all grids, independent of the level. In contrast to the asymptotic rates, the initial convergence rates show large differences for the different grids on a level.

Despite the good and level-independent convergence rates of the $S C M$ process, this is not an efficient method for the recursive application in a multi-level algorithm, because recursive application requires a number of arithmetic operations that is more than proportional to the number of the degrees of freedom. Therefore, in the next section we study an algorithm with a better order of complexity.

\section{Collective correction methods}

\subsection{The plain collective correction method (PCCM)}

In this section we consider methods that solve only a single linear system for each coarse grid on level $\ell-1$ (instead of three). The one solution is used to compute a correction for multiple fine grids. This is implemented in its simplest form in the plain collective correction method (PCCM, see Figure 1). In this algorithm we see several calls for auxiliary routines. The relaxation is damped Jacobi as in Section 4. Because of our interest in a TGM, the coarse grid equations on level $\ell-1$ are solved by a conjugate gradient method. The prolongation of the corrections is done in the routine $P 3 D$. This interpolation is directly based on the hierarchical representation on the levels $\ell-1, \ell-2$ and $\ell-3$, which representation is obtained by restriction from level $\ell-1$ by the scheme given in Figure 2 .

We again study equation (3.1) with homogeneous right-hand side, $f=0$, and initial guess $u_{0}=x(1-x) y(1-y) z(1-z)$. After every cycle with $P C C M$ we calculate the residual and the results are shown in Figure 4. In contrast to the $S C M$ algorithm we see that the convergence behaviour of this algorithm collapses. This is due to non-coherent residuals (or solutions) on the different grids of the finest level, i.e., the residuals on the different grids do not correspond because each grid has its own discretization error. One can even observe that residuals on different grids are equally large, but have opposite signs. Hence the effect of their restriction to the coarse grid is cancelled. This results in a bad convergence behaviour. In the initial phase some grids are converging faster, but the convergence of these particular grids is hampered as they feel the presence of the slowly converging grids. This is also the reason why the residual is not monotonically decreasing for all grids.

\subsection{The Simple Collective Correction Method (SCCM)}

In order to avoid the problem with the non-coherent solutions, as described in Section 5.1 , we simply force the solution to be coherent. This is done in two steps, first changing the function from its redundant representation on level $\ell$ to a hierarchical representation. Second, we transfer the hierarchical representation back to the redundant representation. During the sweep from redundant representation on level $\ell$ to the hierarchical representation, we have to calculate, for the common points on the fine grid, an approximation on the coarse 


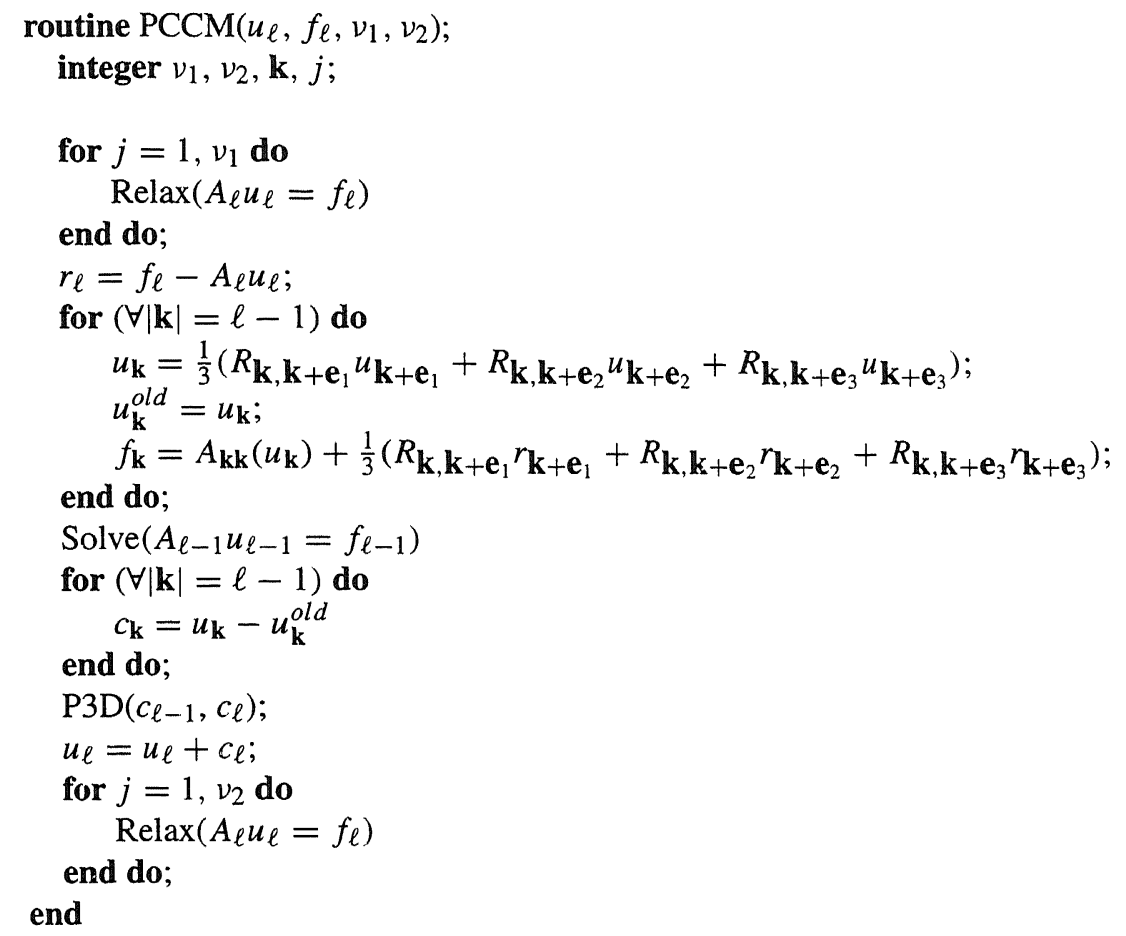

Figure 1. The algorithm PCCM

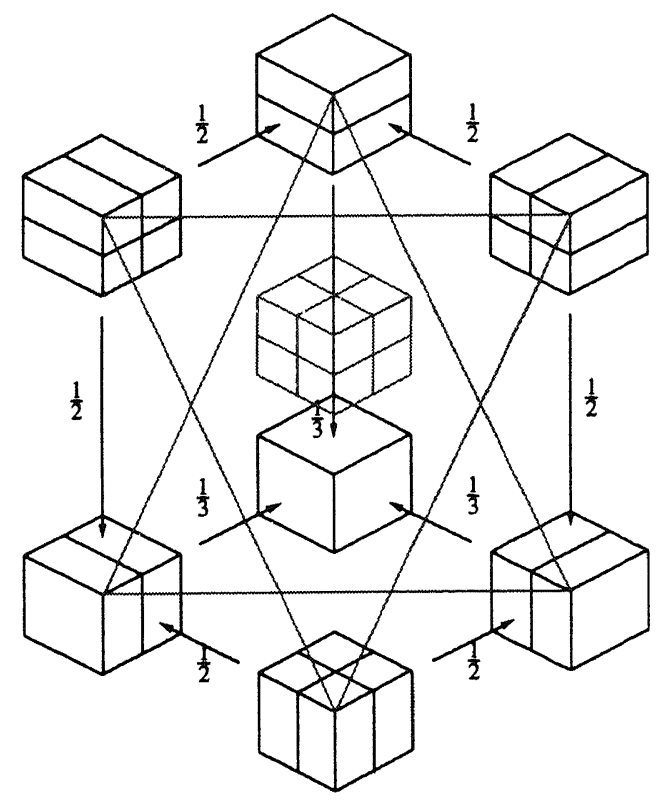

Figure 2. Restrictions of the corrections in $P 3 D$ 

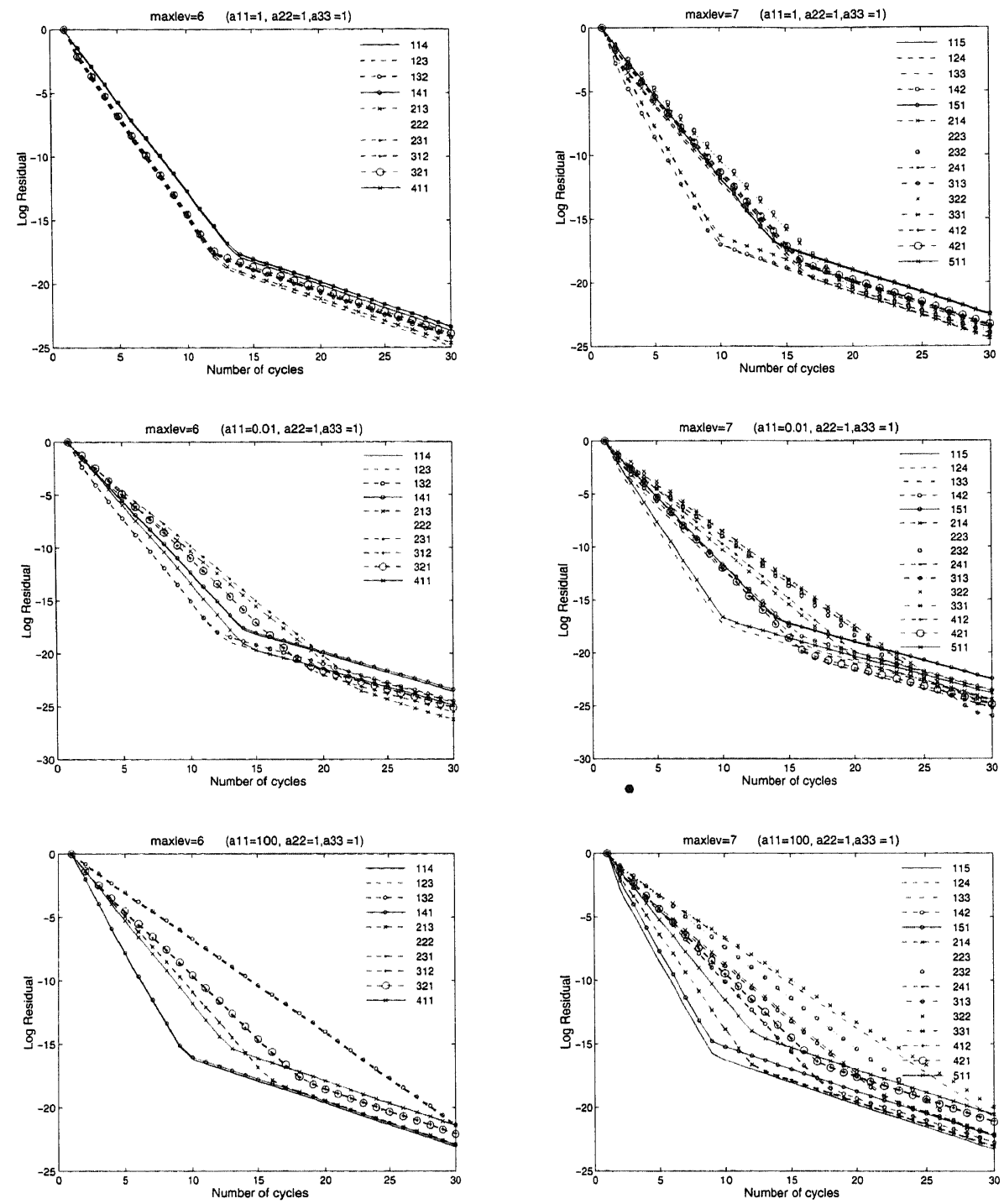

Figure 3. Convergence of $S C M$ for $\ell=6$ (left) and $\ell=7$ (right) for different values of $a_{11}$. The different lines $\left(k_{1}, k_{2}, k_{3}\right)$ indicate convergence histories for different grids on the same level $\ell\left(\ell=k_{1}+k_{2}+k_{3}\right)$ 

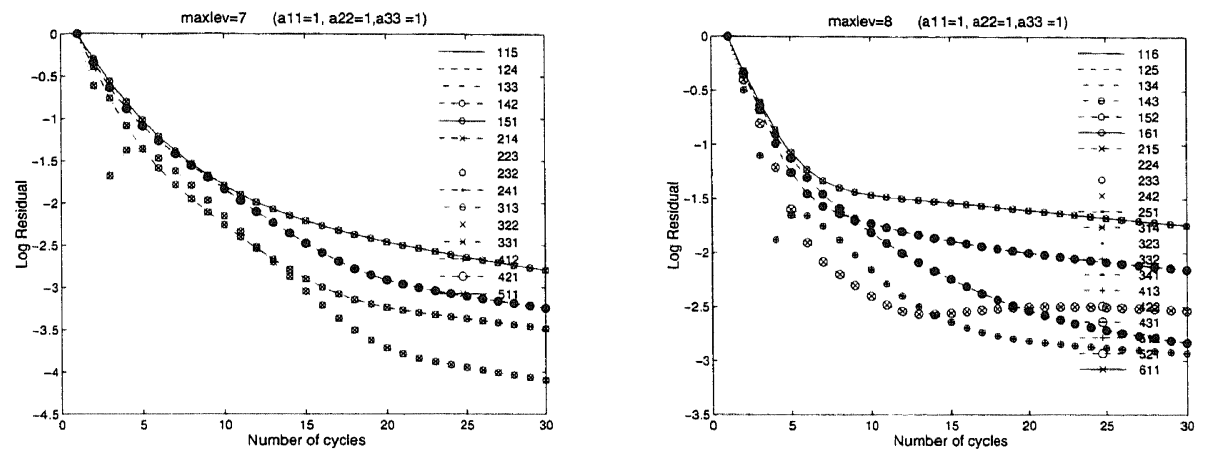

Figure 4. Convergence of $P C C M$ for the trivial solution, for levels $\ell=7$ and $\ell=8$
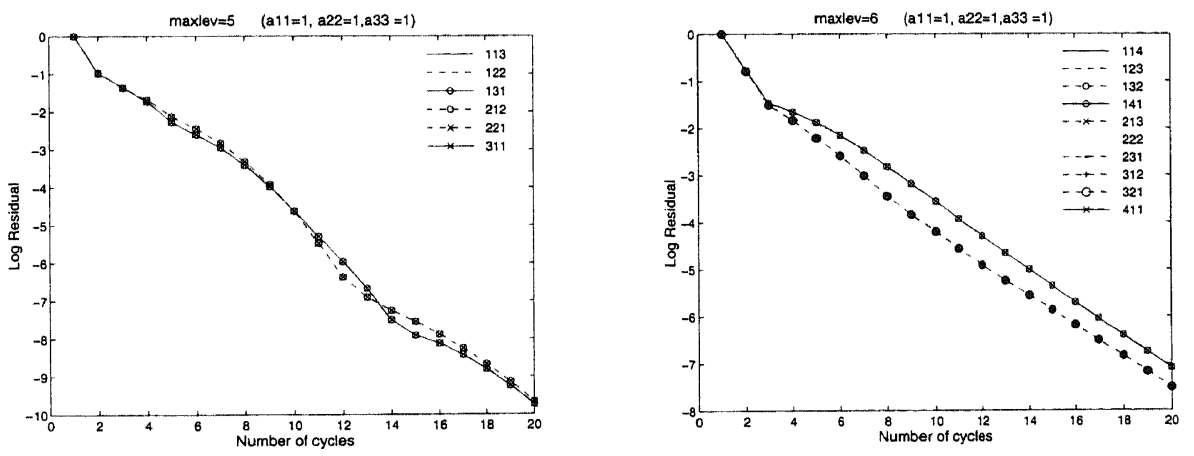

Figure 5. Convergence of $C C M$ for the isotropic equation (3.1), with right-hand side $f=100$, for levels $\ell=5$ and $\ell=6$ 

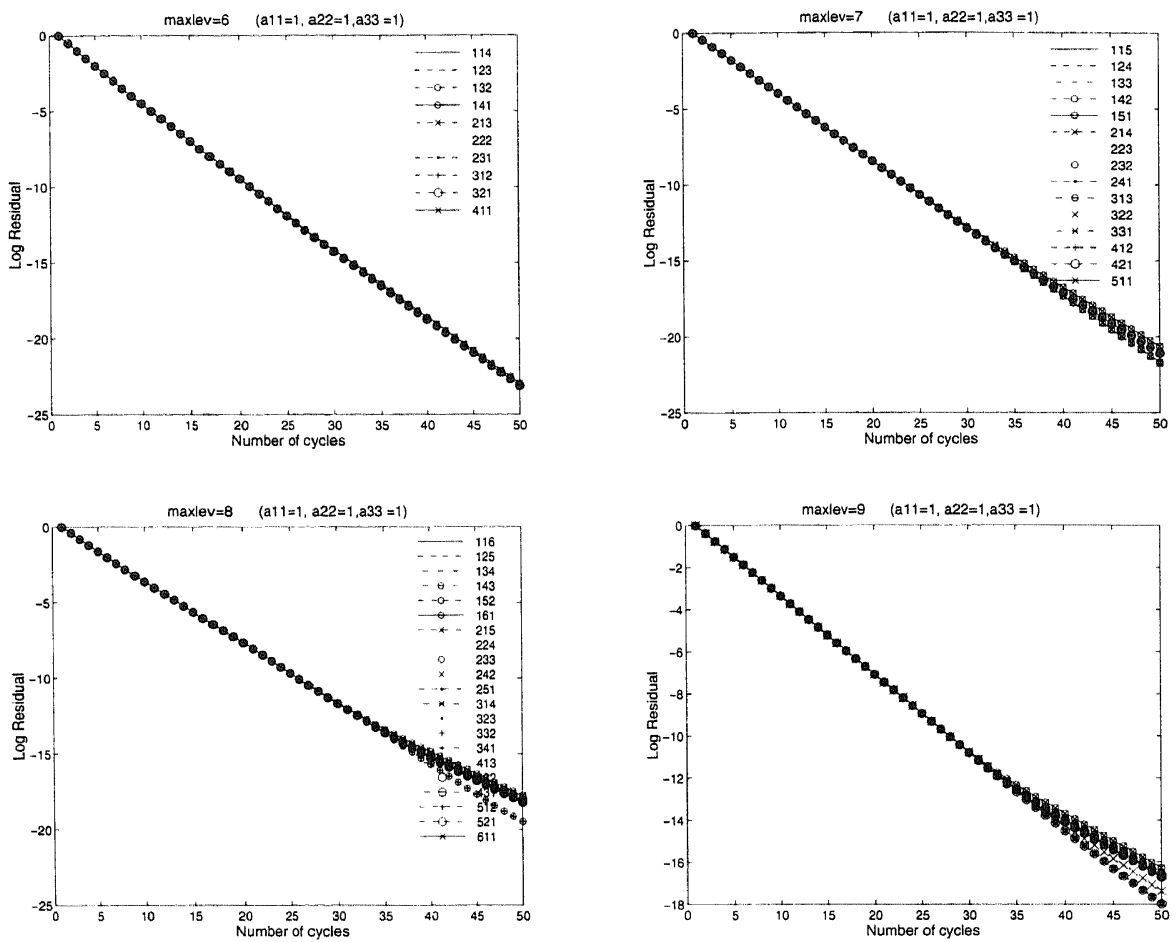

Figure 6. Convergence for the isotropic equation (3.1), for the trivial solution obtained with SCCM

grid. This is done by averaging the three values of the common points on the fine level. This process we call hierarchical smoothing, and in SCCM it is applied after every call for PCCM.

Figure 6 shows the convergence for the isotropic equation (3.1) for various levels. We see that the rate of convergence depends slightly on the level, but the convergence rate is bounded above by a reasonable (constant) value. In Figure 7 we see the convergence results for the anisotropic equation. Again this convergence is independent of the anisotropy.

In our present case, with the trivial solution, we can simply apply hierarchical smoothing to the solution. However, in general, the discrete solutions differ on the different grids, and the hierarchical smoothing is not sufficient. This is due to the fact that the solutions on the different grids all have their own discretization error, so that the discrete solutions do not completely correspond. Hence, when we force the solutions to correspond by hierarchical smoothing we will not obtain a converging solution. Therefore, in the next section we develop a method which is also able to handle non-trivial solutions.

\subsection{The Collective Correction Method (CCM)}

In the previous section we could not expect good convergence rates by SCCM because the solutions on all fine levels differ by their own specific truncation errors. Hence, to obtain 

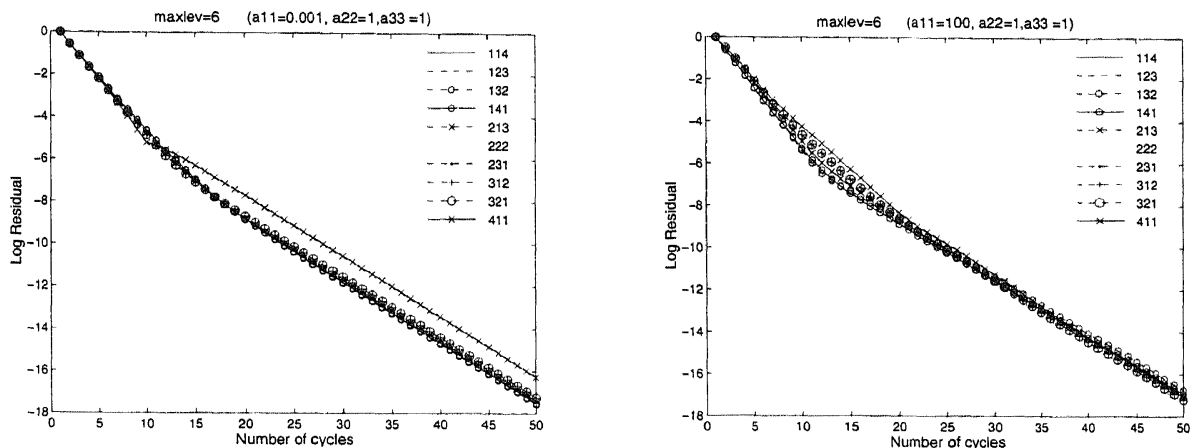

Figure 7. Convergence obtained with $S C C M$, for the anisotropic equation, $a_{11}=0.001$ (left) and $a_{11}=100$ (right), for the trivial solution

corresponding solutions we have to adjust the right-hand side of equation (3.2) for this effect. This leads to the following defect correction equation on each grid $\Omega_{\mathbf{k}}$ :

$$
A_{\mathbf{k} \mathbf{k}} u_{\mathbf{k}}=A_{\mathbf{k} \mathbf{k}} u_{\mathbf{k}}-R_{\mathbf{k} \ell}\left(\tilde{A}_{\ell} \tilde{u}_{\ell}-\tilde{f}_{\ell}\right)=g_{\mathbf{k}}
$$

Here $\tilde{A}_{\ell}$ is some (hierarchical basis) discretization matrix, and $\tilde{u}_{\ell}$ is the coherent representation of the solution, obtained after a sweep of hierarchical smoothing from the redundant representation. The algorithm $C C M$ is obtained from $P C C M$ by replacing the right hand side $f_{\mathbf{k}}$ in (3.2) by the adapted right hand side $g_{\mathbf{k}}$. As in $S C C M$ we apply hierarchical smoothing to the solution.

As we want to obtain good convergence for an arbitrary right-hand side, we consider the non-homogeneous equation (3.1), e.g., with $f=100$. Figure 5 shows the convergence results for the isotropic equation, on levels $\ell=5$ and $\ell=6$. We see that the convergence rate changes somewhat, due to the adjustment of the right hand side. Figure 8 shows the convergence of the anisotropic equation for the levels $\ell=5, \ell=6$ and $\ell=7$. We see that the convergence rate hardly suffers from the anisotropy. Further we see that the convergence is not really dependent on the level of discretization.

\section{Conclusions}

In the asymptotic phase, the convergence rates of the $S C M$ algorithm are almost completely independent of the level of discretization and of the anisotropy of the problem. However, each cycle of this algorithm requires three coarse grid corrections. This implies that the recursive application of this algorithm requires a number of arithmetic operations that is more than proportional to the number of degrees of freedom. Hence, they do not satisfy our aim of an optimal order of complexity. Collective correction algorithms overcome this difficulty.

Good convergence for the collective algorithm depends on coherence between the solutions on the different fine grids. Therefore, we apply hierarchical smoothing to obtain coher- 

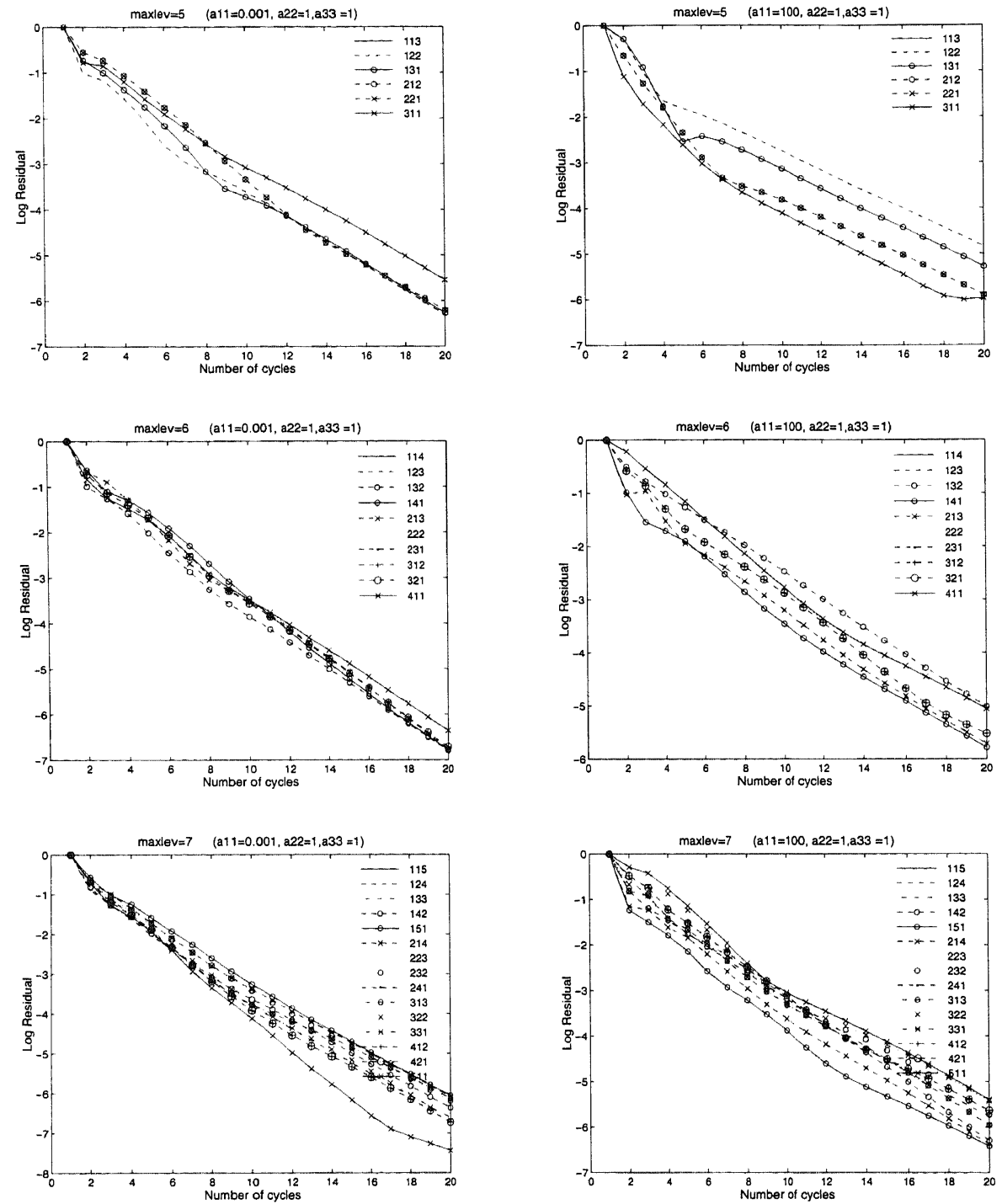

Figure 8. Convergence of $C C M$ for the anisotropic equation (3.1), with $a_{11}=0.001$ (left) and $a_{11}=100.0$ (right) and the right hand side $f=100$, for $\ell=5, \ell=6$ and $\ell=7$ 
ent solutions. Now the solution is solved with a defect correction process that makes use of the hierarchical basis representation. Additionally, we apply the hierarchical smoothing to speed up the convergence of the equations. With this combined process of defect correction and hierarchical smoothing we obtain good convergence results, i.e., convergence which is only slightly dependent on the discretization level and anisotropy of the problem.

Thus far we have calculated the defect correction by means of the hierarchical FE discretization. For the general case this still can be expensive. The next challenge is to find more efficient discretization operators to calculate the defect in (5.1).

\section{REFERENCES}

1. R. Balder and C. Zenger. The solution of multidimensional real Helmholtz equations on sparse grids. SIAM J. Sci. Comput., 17(3), 631-646, 1996.

2. H. J. Bungartz. An adaptive Poisson solver using hierarchical bases and sparse grids. In P. de Groen and R. Beauwens, editors, Proceedings of the IMACS International Symposium on Iterative Methods in Linear Algebra, pages 293-310. IMACS, Elsevier Science Publishers B.V., North-Holland, 1992.

3. H. J. Bungartz. Dünne Gitter und Deren Anwendungen bei der adaptiven Lösung der dreidimensionalen Poisson-Gleichung. Ph.D. thesis, Technische Universität München, 1992.

4. H. Bungartz. and T. Dornseifer. Sparse grids: recent developments for elliptic partial differential equations. Technical Report SFB-Bericht No. 342/02/97, Institut für Informatik, Technische Universität München, 1997.

5. T. Dornseifer. Diskretisierung allgemeiner elliptischer Differentialgleichungen in krummlinigen Koordinatensystemen auf dünnen Gittern. Ph.D. Thesis, Institut für Informatik, Technische Universität München, 1997.

6. M. Griebel. Parallel multigrid methods on sparse grids. Technical Report TUM-I-9053, SFBBericht No. 342/30/90 A, Institut für Informatik, Technische Universität München, 1990.

7. M. Griebel, W. Huber, U. Rüde and T. Störtkuhl. The combination technique for parallel sparse-grid-preconditioning and -solutions of PDEs on multiprocessor machines and workstation networks. Technical Report TUM-I-9214, SFB-Bericht No.342/11/92 A, Institut für Informatik, Technische Universität München, 1992.

8. M. Griebel and P. Oswald. On additive Schwartz preconditioners for sparse grid discretizations. Numer. Math., 66, 449-463, 1994.

9. M. Griebel and P. Oswald. On the abstract theory of additive and multiplicative Schwarz algorithms. Numer. Math., 70, 163-180, 1995.

10. M. Griebel and P. Oswald. Tensor-product-type subspace splittings and multilevel iterative methods for anisotropic problems. Adv. Comp. Math., 4, 171-206, 1995.

11. M. Griebel, M. Schneider and C. Zenger. A combination technique for the solution of sparse grid problems. Technical Report TUM-I-9038, SFB-Bericht No. 342/19/90 A, Institut für Informatik, Technische Universität München, 1990.

12. P. W. Hemker. Sparse-grid finite-volume multigrid for 3D-problems. Adv. Comp. Math., 4, 83-110, 1995.

13. P. W. Hemker and C. Pflaum. Approximation on partially ordered sets of regular grids. App Numer. Math., 25, 55-87, 1997.

14. B. Koren, P. W. Hemker and P.M. de Zeeuw. Semi-coarsening in three directions for Eulerflow computations in three dimensions. In H. Deconinck and B. Koren, editors, Euler and Navier-Stokes Solvers Using Multi-dimensional Upwind Schemes and Multigrid Acceleration, volume 57 of European Community Research in Aeronautics, Notes on Numerical Fluid Mechanics, pages 547-567, Braunschweig, Vieweg, 1997.

15. C. B. Liem, T. Lü and T. M. Shih. The Splitting Extrapolation Method. World Scientific, Singapore, 1995.

16. W. A. Mulder. A new multigrid approach to convection problems. J. Comp. Phys., 83, 303-323, 1989.

17. W.A. Mulder. A high-resolution Euler solver based on multigrid, semi-coarsening, and defect 
correction. J. Comp. Phys., 100, 91-104, 1992.

18. G. Niessen. On the stability of sparse grid splitting. Technical Report No. 115, Institut für Geometrie und Praktische Mathematik, RWTH-Aachen, 1995.

19. C. Pflaum. A multilevel algorithm for the solution of second order elliptic differential equations on sparse grids. In N. D. Melson, T. A. Manteuffel, S. F. McCormick and C. C. Douglas, editors, Seventh Copper Mountain Conference on Multigrid Methods, volume CP 3339, Hampton, VA, pages 661-672, NASA, 1996.

20. P. S. Vassilevski. On two ways of stabilizing the hierarchical basis multilevel methods. SIAM Rev., 39, 18-53, 1997.

21. C. Zenger. Sparse grids. Technical report, Institut für Informatik, Technische Universität München, 1990. 